\title{
La deserción universitaria: resiliencia como posibilidad de logro
}

\author{
María Paula Seminara
}

\begin{abstract}
Resumen
La deserción universitaria emerge como un fenómeno multifactorial alarmante porque presenta consecuencias en diferentes planos y para distintos actores: estudiantes, instituciones y el Estado. La literatura da cuenta de lo frecuente que resulta poner el acento en el estudiante como responsable casi exclusivo de sí mismo, a la vez que invita a cuestionar la persistencia de esta problemática aun tras décadas de investigación y de múltiples estrategias implementadas para abordarla. Éste y otros cuestionamientos evidencian la necesidad de un cambio de perspectiva para no perder de vista el papel que juegan las instituciones en lo que sucede respecto a la deserción universitaria, así como la urgencia de revisar las concepciones de éxito y fracaso normalizadas dentro del ámbito estudiantil de las universidades. Examinar estas ideas e interpelar a las instituciones puede ser uno de los caminos para encontrar alternativas frente a una mirada de este fenómeno que se considera determinista. El presente artículo explora la resiliencia como factor clave en la posibilidad de logro y enfatiza la importancia de observar las potencialidades de los estudiantes para abordar el fracaso y conducirnos hacia una universidad más democrática.
\end{abstract}

Palabras clave: deserción universitaria, resiliencia, concepciones de éxito/logro, fracaso.

\section{University Dropout: Resilience as a Possibility of Achievement}

\begin{abstract}
Dropping out of university is an alarming multifactorial phenomenon because it affects different levels and different agents: students, institutions and the State. Specialized literature suggests that it is common to highlight the student as the sole responsible for quitting superior levels of education. In addition, it poses questions on the persistence of this phenomenon even after decades of research and multiple strategies implemented to address it. It is evident that a change of perspective is needed to look at the role played by institutions within this complex scheme, as well as the urgency to review the normalized notions of success and failure among students at university. Examining these ideas and challenging institutions can be one of the ways to find alternatives to a social phenomenon otherwise regarded as deterministic. This article explores resilience as a key factor in the possibility of success and emphasizes the importance of looking at the students' potential to address failure and, eventually, lead us towards a more democratic university.
\end{abstract}

Keywords: university dropout, resilience, conceptions of success/achievement, failure.

Recepción: 30/07/2019. Aceptación: 20/05/2020

Dol: http://doi.org/10.22201/cuaieed.16076079e.2020.21.5.11 


\section{María Paula Seminara}

paulaseminaratorcivia@gmail.com

Lic. En Psicología Universidad Nacional de Córdoba, Argentina. Doctorando en Ciencias de la Educacion por la Universidad Católica de Cuyo, Argentina. Becaria Doctoral Consejo Nacional de Ciencia y Técnica, Argentina. Jtp Facultad de Ingenieria, Universidad Nacional de San Juan, Argentina.

\section{Introducción}

La universidad moderna se había tratado más de un privilegio que de un servicio que ve por la formación de sus estudiantes, pues ha atendido a una exclusiva minoría como la de los herederos ${ }^{1}$ que describieron Bourdieu y Passeron (1970). Se ha llegado a considerar a la universidad como una institución de élite por el número reducido de estudiantes que solía recibir y por la extracción social de los mismos, tal y como explica Trow (Brunner, 2014). Tras la masificación de la universidad, surgen efectos como la incorporación a la educación superior de estudiantes no tradicionales, así como la cronicidad ${ }^{2}$ y la deserción. Estas problemáticas desestabilizan las nociones de éxito y fracaso en la universidad que, una vez normalizadas, matizan los condicionantes socioeconómicos de los alumnos bajo la idea de mérito propio o de selección natural a partir de ciertas aptitudes innatas. Revisar los criterios de éxito implica debatir si se trata de características fijas de los estudiantes o, por el contrario, si los condicionantes inherentes al alumno son desarrollables. Considerar que hay factores

${ }^{1}$ Con el término de herederos, Bourdieu y Passeron aludían a una minoría de estudiantes privilegiados que accedían a la Educación Superior, la cual tendía así a ser reproductora de las clases sociales.

${ }^{2}$ La cronicidad refiere a la demora de los estudios respecto a lo que estipula la trayectoria teórica planteada por los planes de estudio, mientras que la deserción refiere al abandono, transitorio o definitivo, de los estudios. intervinientes que pueden aprenderse y dirigirse hacia la posibilidad de logro, o incluso propiciarse en el ámbito institucional, conduce a la resiliencia como factor posibilitador.

En la primera parte de este escrito se alude a la etimología de las concepciones sobre éxito y fracaso, así como a su normalización e implicaciones en la universidad. En la segunda, se revisa el concepto de resiliencia como una bisagra que se bifurca entre fracaso y éxito. El tercer eje articula la resiliencia con el fenómeno de deserción y las concepciones revisadas; además, se planta una visión alternativa a la que, con frecuencia, se observa en la literatura y que amplía la perspectiva hacia las potencialidades en pos de la permanencia en nuestras universidades. 


\section{Eje 1. Concepciones de éxito y fracaso, y sus implicaciones en la universidad}

El éxito y el fracaso son fenómenos multifactoriales difíciles de definir y con variadas implicaciones. Podría hipotetizarse que existe cierta reproducción de éxitos y fracasos, a partir de rasgos y eventos particulares que emergen desde el ingreso a la universidad y van creciendo exponencialmente a lo largo del tiempo. Dicho de otro modo, los fracasos o éxitos iniciales, que dependen de condiciones socioeconómicas, de la preparación en la escuela media y de otros factores, parecen ir retroalimentándose en forma de espiral. Esto ocurre de modo que quienes ingresan con menores herramientas ven sus trayectorias obstaculizadas y terminan por rezagarse en el camino o, en el peor escenario, abandonando su educación superior.

Dentro del fracaso, algunos autores cuestionan si la deserción escolar se trata de una evasión o una expulsión por parte del sistema (Rojas, 2009; de Vries et al., 2011). A continuación se retoman un par de preguntas que redirigen la responsabilidad del sistema de educación superior por la pérdida de estudiantes: ¿abandonar es una decisión del alumno? y ¿el ingreso ilimitado implica equidad para todos?

La deserción es la desafiliación del alumno del sistema, de forma voluntaria o forzosa, definitiva o transitoria, y puede presentarse en relación a una carrera o institución particular, así como al sistema en su conjunto. No todos los tipos de abandono merecen igual intervención, ni conllevan el mismo sello negativo. Entre las causas existen aquellas de índole individual como el género, la integración social, o la edad; socioeconómicas, como el estrato social, la situación laboral del estudiante y sus padres; académicas, por ejemplo, la orientación vocacional, rendimiento académico, métodos de estudio; o institucionales, normativas que incluyen relación pares y profesores, infraestructura, entre otras (Aparicio, 1998). La literatura revela numerosos estudios que no han hallado aún el punto que persiste sobre el fenómeno (Seminara y Aparicio, 2018).

Se trata de ver, además de las causas superficiales, las representaciones que le son asociadas a los términos en cuestión. La palabra éxito, de procedencia latina, quiere decir salida. La palabra fracaso hace referencia a la frustración cuando se malogra una pretensión y proviene del vocablo italiano fracassare, que puede traducirse como estrellarse o romperse (Diccionario etimológico en línea, 2020). Cabe preguntar: ¿quién fracasa en la deserción? ¿Qué se considera que es ser un alumno exitoso? ¿Quién califica el éxito o fracaso estudiantil como tal? ¿Se trata de características determinadas e inamovibles? ¿Cómo puede intervenir la institución para cambiar el desenlace de quienes fracasan? ¿Qué características, si las hay, son propias de quienes persisten? ¿Pueden desarrollarse en quienes desertan? ¿Qué mirada alternativa al determinismo puede contribuir a la permanencia de quienes parecen destinados al fracaso en el sistema? 
Se han revisado investigaciones de González Lizárraga, López González y Parra Ramos (s.f.) que indican variables asociadas al éxito en estudiantes y, en este sentido, Powell, Conway y Ross (1990) consideran que el éxito está vinculado a la finalización de los estudios, al igual que Cuellar Martínez (2003). En contraste, de los Santos (2000) añade que el éxito consiste en finalizar los estudios en los tiempos estipulados por los planes de estudio. Otros investigadores agregan que no se trata únicamente de terminar, sino hacerlo con calificación sobresaliente. Stelnicki, Nordstokke y Saklofske (2015) se ocupan de reportar las percepciones de los estudiantes sobre las fortalezas y debilidades que contribuyen a su éxito durante la carrera de grado. Entre las fortalezas, mencionan la orientación clara de futuro, la persistencia y la organización. Los resultados demuestran que el estrés, las habilidades académicas inadecuadas y las distracciones son detrimentos para el éxito universitario. Bonneville-Roussy et al. (2017) probaron un modelo que vinculaba motivación, afrontamiento de las dificultades y resultados académicos en la universidad, y mostraron que afrontamientos menos comprometidos fueron vinculados a resultados menos adaptativos y que lo opuesto sucedía con la motivación autodeterminada o intrínseca y el afrontamiento activo.

Según Perrenoud (2008, cit. por Valle et al., 2014), un alumno exitoso es quien cumple con las expectativas de la institución educativa al interior de la cual se fabrican los juicios de excelencia a los que no puede sustraerse. Es decir, que el éxito o fracaso académico son el resultado de juicios más bien automáticos (Valle et al., 2014) y el juicio de la institución influye en la construcción del juicio que el alumno hace sobre sí mismo. Entre muchos trabajos, resulta evidente que las percepciones de éxito y fracaso y la motivación y el modo de afrontar las exigencias de la carrera inciden de manera notable en el resultado (Valle et al., 2014; Rovella y Sans, 2005). Entre las preguntas posibles, aquí se destacan si estas percepciones pueden modificarse, si las instituciones efectivamente intervienen y qué modelo conciben para aportar estrategias acordes a la realidad de la problemática.

Hay varias posturas frente a la posición anteriormente hipotetizada. Así, González Fiegehen (2006) plantea que el rezago es una alerta que puede derivar en deserción, en tanto Tinto (1989) dice que quienes se retrasan pueden ser los que más persisten (Losio y Macri, 2015). Lo cierto es que, como postulan Salanova Soria et al. (2005), existen facilitadores y obstaculizadores del bienestar estudiantil asociados a la permanencia que también presentan una positiva correlación entre éxito o fracaso pasados, bienestar o malestar psicológico y éxito o fracaso futuros. Por lo expuesto, se entiende que el logro y el fracaso no son acontecimientos con proyecciones lineales y no dependen sólo de características de los alumnos sino de factores institucionales (Valle et al., 2014). Se sugiere que la sumatoria de eventos exitosos o de fracaso conduce a círculos viciosos o virtuosos en las trayectorias educativas y que el desarrollo de la resiliencia podría conducir a un egreso exitoso. A su vez, que las instituciones pueden generar espacios posibilitadores de resiliencia (Henderson y Milstein, 2003) o entornos 
Video 1. Éxito y fracaso, ¿dos caras de una misma moneda? Por Diego Pasjalidis (TEDx Talks, facilitadores, pues se trata de dejar de concebir el éxito académico como mérito de aquellos con características aparentemente privilegiadas, para que así se abra la universidad no sólo con el acceso, sino también favoreciendo la permanencia. Por el momento, y lejos de suponer que ya no hay espacio de debate, cabe señalar que las nociones de éxito y fracaso en un contexto escolar son conceptos complejos o, como señala Diego Pasjalidis, dos caras de una misma moneda (ver video 1).

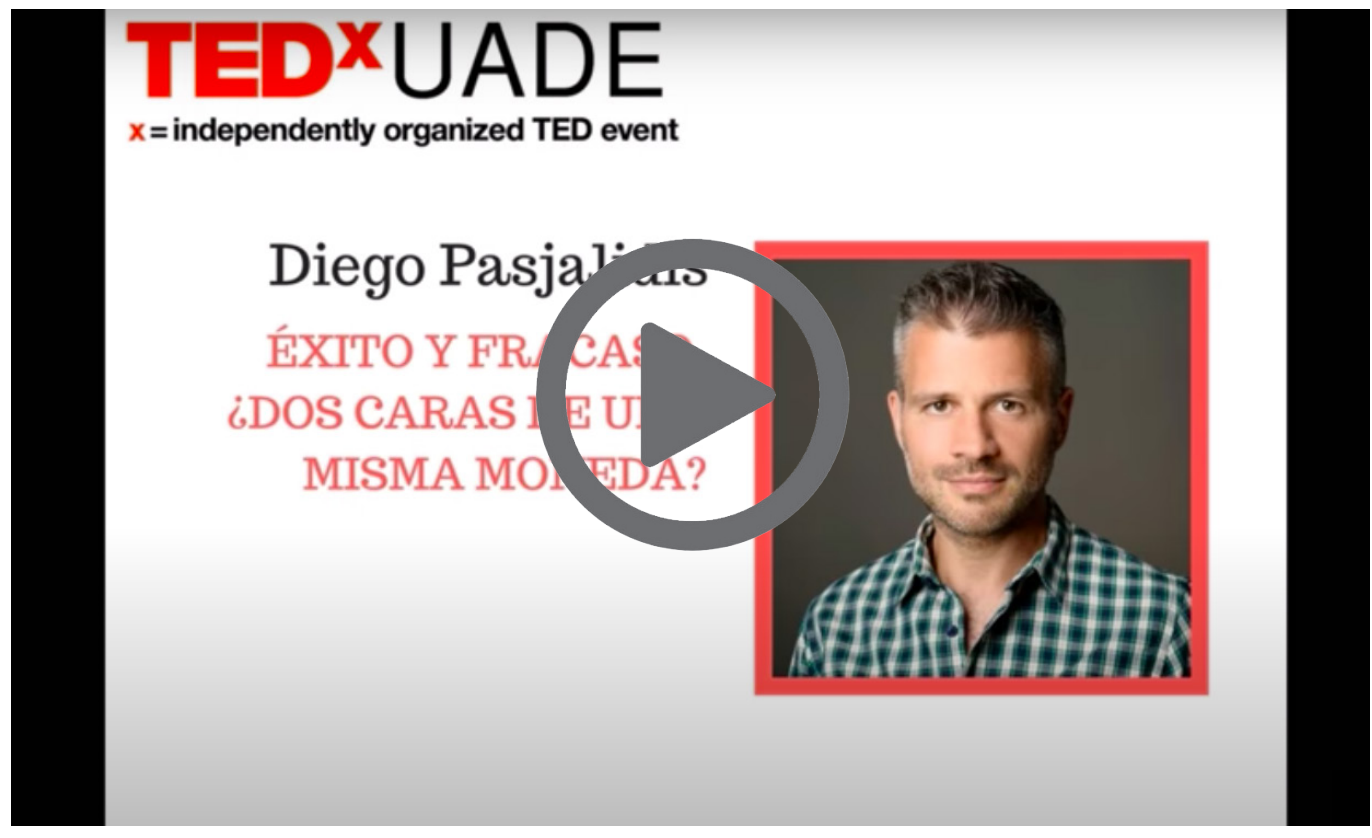

\section{Eje 2. Recuperar el rol de la resiliencia como clave ante la deserción}

Tradicionalmente, la psicología se centró en la psicopatología y en la descripción exhaustiva de enfermedades (Kotliarenco, Cáceres y Fontecilla, 1997). No obstante, muchas predicciones de resultados negativos en función de factores de riesgo no se cumplían (Werner, 1989 cit. por Coronado-Hijon, 2017). A fines de 1990, el psicólogo M. Seligman sistematizó los estudios de los aspectos más saludables de los individuos, y así creo una nueva corriente que denominó psicología positiva. Forma parte del cambio de enfoque desde lo patológico hacia lo saludable, y busca brindar herramientas para lograr una mejor calidad de vida y superar los obstáculos que la dificultan (Lorenzo, 2010). Adopta el concepto de resiliencia, aludiendo a que, mientras el estrés se resiste a la novedad, ésta afronta el problema (Wolin y Wolin, 1993 cit. por Henderson y Milstein, 2003).

Según la Organización Mundial de la Salud (Munist et al., 1998), la palabra resiliencia se usa en metalurgia e ingeniería para describir la capacidad de ciertos materiales de recobrar su forma original después de sufrir una presión deformadora. A partir de los años sesenta del siglo pasado, el término fue 
adoptado por las ciencias sociales para caracterizar a quienes, a pesar de vivir en condiciones de riesgo, se desarrollaban psicológicamente sanos y socialmente exitosos (Block y Kremen, 1996). Mientras que en física se habla de volver a un estado anterior, en psicología el concepto se amplía, ya que, en realidad, no se vuelve a un estado inicial, sino que el aparato psíquico se reorganiza aún de manera más eficiente (Lorenzo, 2010).

García-Vesga y Domínguez-de la Ossa (2013) identifican dos momentos en la evolución del concepto. En el primero, se analizaban factores individuales protectores y, en el segundo, se reconocieron ambientes donde las personas se desenvuelven como factores de protección. En otras palabras, se pasó de lo personal a lo relacional. Según Werner (1993, cit. por Meisels y Shonkoff, 2000), Ios factores protectores operan a través de tres mecanismos: compensar el estrés por cualidades o fuentes de apoyo, tomarlo como desafío si no es excesivo, y tomarlo como inmunidad. De esta manera se sugiere que los factores protectores modulan el impacto del estrés en calidad de adaptación. Por otra parte, los factores protectores pueden ser internos-personales o externos, que refieren a condiciones del medio que reducen la probabilidad de daños como la familia o la integración social.

La resiliencia, por tanto, es resultado de la armonía entre factores de riesgo y factores protectores internos y externos. Así, la persona puede "estar" más que "ser" resiliente, en el entendido de que la resiliencia no es una cualidad verificable en to do momento. Desde este enfoque, se la concibe como un proceso dinámico, dependiente de factores internos y externos que en interacción con el riesgo hacen posible enfrentar situaciones adversas. En la misma línea, Cyrulnik (2001, 2009 cit. por Melillo, 2005) destaca que no hay un comienzo absoluto del proceso resiliente ni una delimitada capacidad de resiliencia. Entenderla como proceso permite inferir que puede desarrollarse. Y es que, durante las crisis de la vida (como es el tránsito por la universidad), su promoción marca la diferencia entre el desarrollo de las potencialidades o el estancamiento (Saavedra Guajardo y Villalta Paucar, 2008).

No obstante, el estudio de la resiliencia en instituciones educativas ha sido pobre, especialmente en las de educación superior (Haz y Castillo, 2003; Lamas y Lamas, 1997; Lightsey, 2006, cit. por Peralta Díaz; Ramírez Giraldo y Castaño Buitrago, 2006). Más aún, Henderson y Milstein (2003) afirman que la mayoría de las investigaciones se ha centrado en niños y adolescentes. Los autores elaboraron un modelo de seis pasos que promociona la resiliencia en contextos formales, poniendo el foco en la adquisición y desarrollo de competencias, y no en el déficit. El modelo consiste en:

1. Brindar afecto y apoyo como sostén del éxito académico.

2. Establecer y transmitir expectativas elevadas y realistas.

3. Brindar oportunidades de participación significativas. 
4. Enriquecer los vínculos con un sentido de comunidad educativa.

5. Dar participación a toda la comunidad.

6. Enseñar habilidades para la vida, cooperación, resolución de conflictos, destrezas comunicativas, habilidad para resolver problemas y tomar decisiones. Implica una posición respecto a la democratización de la educación superior.

Según Casassus (2005, cit. por Montes y Parcerisa, 2016) el sistema educativo puede reproducir, mantener o reducir las desigualdades; lo que equivale a decir que, independientemente de las fuerzas que se transmiten desde la estructura social (al interior del sistema), se encuentran poderosos mecanismos de diferenciación social. Brindar herramientas de desarrollo ante las adversidades contribuye a la equidad que va más allá de la igualdad.

\section{Eje 3. Incluir la resiliencia para mirar la deserción: entre el fracaso y la posibilidad de éxito}

Pascarella y Terenzini (2005, cit. por Aparicio, 1998 y Reason, 2009) exponen recomendaciones para avanzar en la comprensión de cómo la universidad afecta a los estudiantes, tales como reconocer la creciente diversidad de estudiantes, expandir la investigación sobre estudiantes e instituciones previamente ignorados, entre otros. Considerando estas recomendaciones, la resiliencia y el ingreso de estudiantes no tradicionales a la universidad (como estudiantes de medio tiempo que trabajan y/o tienen familia a cargo), a continuación, se presenta una mirada alternativa sobre la deserción universitaria (ver figura 1).

Figura 1. Mirada alternativa de la deserción desde la resiliencia (Díaz Peralta, 2008; Salanova Soria et al., 2005, entre otros). Fuente: elaboración propia.

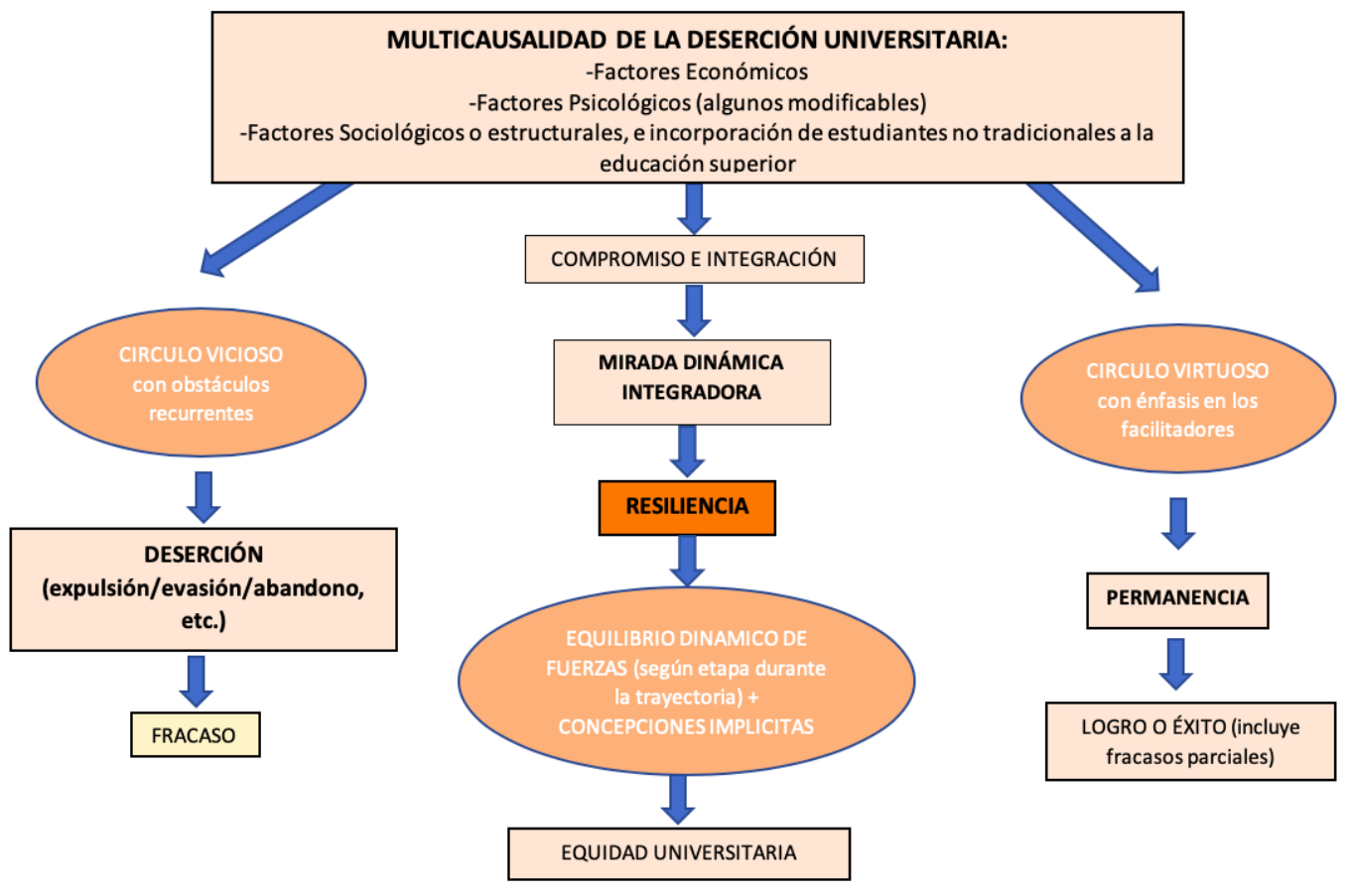


El esquema presenta una mirada dinámica que sostiene la multicausalidad de la deserción universitaria. En él, los condicionantes indirectos influyen sobre el grado de compromiso e integración social y académica del estudiante en la institución universitaria. Asimismo, los condicionantes se presentan en combinaciones diferentes según la etapa de la trayectoria en la que se encuentre el estudiante. Éstos tienen mayor preponderancia en algunos momentos, pero todos afectan de manera constante. Esta distinción por etapas permite entender que las estrategias de retención deben ser diferentes según hablemos de ingresantes o alumnos avanzados.

A las concepciones de éxito y fracaso se suman otros factores influyentes que se mantienen en un equilibrio de fuerzas que el estudiante y la institución deben manejar y que pueden desencadenar círculos virtuosos hacia el logro. También se pueden desencadenar círculos viciosos por fracasos repetidos y que tengan como desenlace la permanencia o la deserción (Díaz Peralta, 2008). Ante la posible reproducción de éxitos y fracasos, la resiliencia podría resultar clave para virar hacia el logro aquellas trayectorias adversas. En este sentido, es importante apuntar a desarrollar el proceso de resiliencia y a crear un contexto institucional que la favorezca.

\section{Conclusiones}

Al inicio se planteó la siguiente pregunta: ¿por qué persiste el fenómeno de desercón luego de décadas de investigación y de múltiples estrategias implementadas para abordarlo? Este trabajo mostró a la deserción como fenómeno multicausal de responsabilidad compartida. En la primera parte, se aludió a las concepciones de éxito y fracaso y sus implicaciones en la universidad. En la segunda, se enfatizó en el concepto de resiliencia como clave y bisagra posible entre el fracaso y el éxito. En la tercera, se presentó una mirada alternativa (no determinista) de la deserción, enfatizando la importancia de incluir la resiliencia. Se intenta explicar que las causas fungen de manera distinta según el período de la trayectoria y que se trata de apostar por las potencialidades de los estudiantes para no quedarnos en una concepción de la educación como supervivencia meritocrática. Para ello hay que entender los factores que viran el fracaso hacia el éxito, o como dice Carlos Égido: "El que resiste, gana” (ver video 2).

Por último, se expuso que se trata no sólo de estudiar las causas de la deserción, sino de buscar las posibilidades de permanencia en un intento por lograr una verdadera democratización de la educación superior. Así, cabe tener presente algunas preguntas del tipo: ¿propiciamos desde la universidad el despliegue de las potencialidades de los alumnos? y ¿trabajamos por la equidad que abunda en los discursos que proferimos? En resumen, ¿hacemos cotidianamente una institución verdaderamente universitaria? Y es que el cambio necesario no está sólo en grandes hallazgos, sino en revisar lo que hacemos a diario, cuestionarlo y trabajar por su mejora. 
Video 2. El que resiste, gana por Carlos Egido (TEDx Talks, 2018).

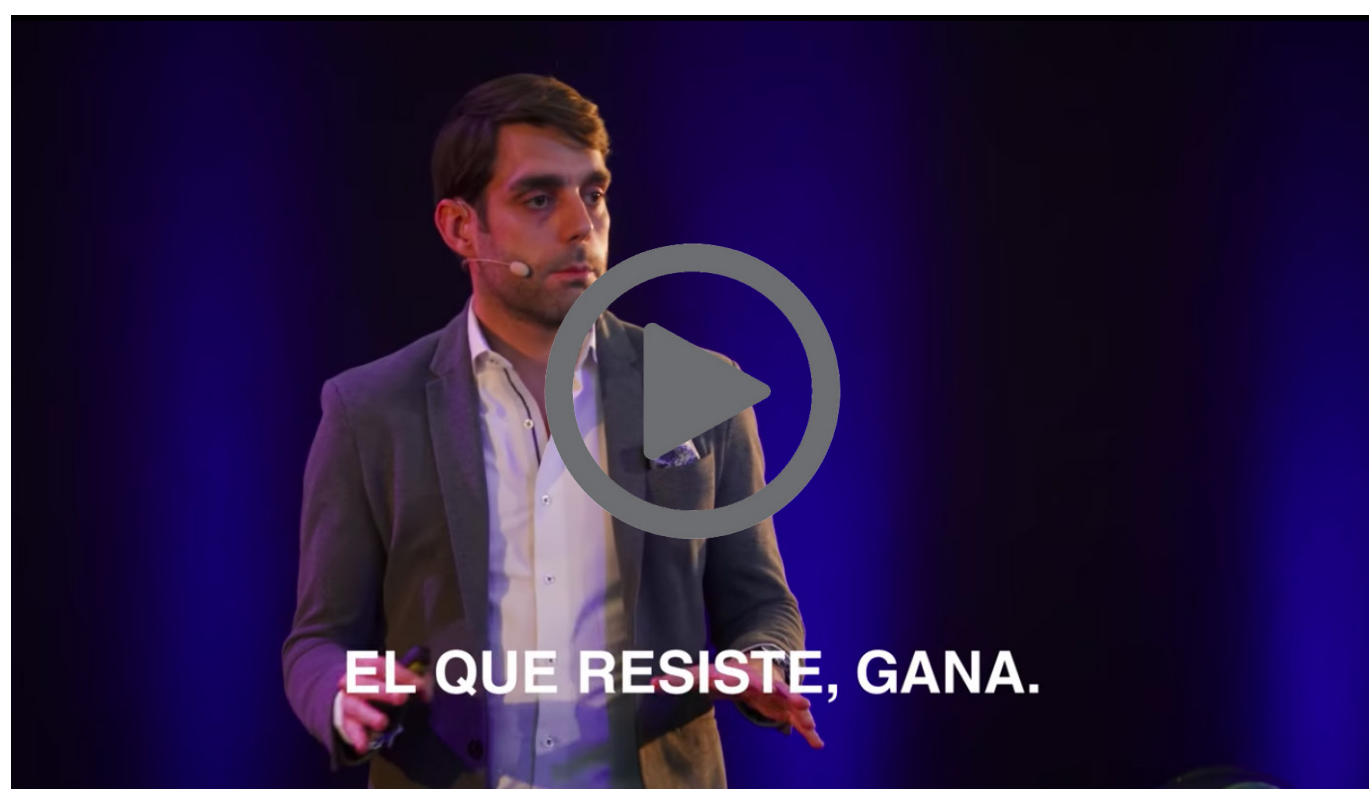

\section{Referencias}

* Aparicio, M. (1998). Causas de la Deserción en Universidades Nacionales. Universidad Nacional de Cuyo.

* Block, J., y Kremen, A. (1996). IQ and ego-resiliency: Conceptual and empirical connections and separateness. Journal of Personality and Social Psychology, 70(2), 349-361. Dol: https://doi.org/10.1037//0022-3514.70.2.349

* Bonneville-Roussy, A., Evans, P., Verner-Filion, J., Vallerand, R. J., y Bouffard, T. (2017, enero). Motivation and coping with the stress of assessment: Gender differences in outcomes for university students. Contemporary Educational Psychology, 48, 2842. Dol: https://doi.org/10.1016/j.cedpsych.2016.08.003

* Brunner, J. (2014). Universidad, poder y derecho. Páginas de Educación, 7(2), 06-32. http://www.scielo.edu.uy/pdf/pe/v7n2/v7n2a02.pdf

* Coronado-Hijon, A. (2017). Academic resilience: A transcultural perspective. Procedia. Social and Behavioral Sciences, 237, 594-598. Dol: https://doi.org/10.1016/j. sbspro.2017.02.013

* De Vries, W., León Arenas, P., Romero Muñoz, J., y Hernández Saldaña, I. (2011, octubre-diciembre). ¿Desertores o decepcionados? Distintas causas para abandonar los estudios universitarios. Revista de la Educación Superior, xL(4)(160), 29-49. https://www.redalyc.org/pdf/604/60422569002.pdf

* Díaz Peralta, C. (2008). Modelo conceptual para la deserción estudiantil universitaria chilena. Estudios pedagógicos, xxxiv(2), 65-86. Dol: http://dx.doi. org/10.4067/S0718-07052008000200004

* Diccionario etimológico en línea. (2020). http://etimologias.dechile.net 
* Franco, A., Blanca, M., Cardona Marín, Á., y Molina Velásquez, D. (2008, septiembrediciembre). Situaciones conflictivas que propician las transformaciones familiares. Revista Virtual Universidad Católica del Norte, 25. http://www.redalyc.org/articulo. oa?id=194215513011

* García-Vesga, M. C., y Domínguez-De La Ossa, E. (2013). Desarrollo teórico de la Resiliencia y su aplicación en situaciones adversas: Una revisión analítica. Revista Latinoamericana de Ciencias Sociales, Niñez y Juventud, 11(1), 63-77. https://www. redalyc.org/articulo.oa?id=773/77325885001

* González Fiegehen, L. (2006). Repitencia y deserción universitaria en A. L. Informe sobre Educación Superior en América Latina y el Caribe 2000-2005. Metrópolis.

* González Lizárraga, G., López González, R., y Parra Ramos, M. E. (s.f.) ¿Éxito o Fracaso académico en la Universidad Sonora? http://www.comie.org.mx/ congreso/memoriaelectronica/v09/at16/PRE1178908763.pdf

* Henderson, N. Y., y Milnstein, M. (2003). Resiliencia en la escuela. Paidós.

* Kotliarenco, M. A., Cáceres, I., y Fontecilla, M. (1997). Estado de arte en resiliencia [base de datos en línea]. Estados Unidos: Organización Panamericana de la Salud, Oficina Sanitaria Panamericana, Oficina Regional de la Organización Mundial de la Salud. http://www1.paho.org/Spanish/HPP/HPF/ADOL/Resil6x9.pdf

* Lorenzo, R. (2010). Resiliencia. Nuestra capacidad de recuperación ante los obstáculos. Andrómeda.

* Losio, M., y Macri, A. (2015, noviembre). Deserción y rezago en la universidad. Indicadores para la Autoevaluación. Revista Latinoamericana de Políticas y Administración de la Educación, 2(3), 114-126. https://www.uai.edu.ar/media/42456/ ganadores-2016_deserci\%C3\%B3n-y-rezago-en-la-universidad.pdf

* Meisels, S., y Shonkoff, J. (2000). Handbook of Early Child Intervention. Cambridge University Press.

* Melillo, A. (2005). El pensamiento de Boris Cyrulnik. Perspectivas Sistémicas, 85. http://www.redsistemica.com.ar/melillo.htm

* Montes, A., y Parcerisa, L. (2016) Iguales en qué y cómo. Una revisión de las propuestas realizadas desde la teoría de la justicia al campo de la educación. Revista de sociología, 101(4), 451-471. http://dx.doi.org/10.5565/rev/papers.2194

- Munist, M., Santos, H., Kotliarenco, M. A., Suárez Ojeda, E. N., Infante, F. Y Grodberg, E. (1998, septiembre). Manual de resiliencia en niños y adolescentes. Organización Panamericana de la Salud; Organización Mundial de la Salud. https://es.scribd. com/document/47568179/OMS-Manual-Sobre-Resiliencia

- TEDx Talks. (2016, 1 de diciembre). Éxito y fracaso, ¿Dos caras de una misma moneda? | Diego Pasjalidis | TEDxUADE [video]. YouTube. https://youtu.be/X5Aags8GEpg

* TEDx Talks. (2018, 15 de agosto). El que resiste, gana | Carlos Egido I TEDxHumboldtMexicoCity [video]. YouTube. https://youtu.be/cqQVJCHBMOY 
* Peralta, S., Ramírez, A., y Castaño, H. (2006). Factores resilientes asociados al rendimiento académico en estudiantes pertenecientes a la Universidad de Sucre (Colombia). Psicología desde el Caribe, 17, 196-219. https://www.redalyc.org/ articulo.oa?id=21301709

* Reason, R. (2009, noviembre-diciembre). An Examination of Persistence Research Through the Lens of a Comprehensive Conceptual Framework. Journal of College Student Development, 50(6), 659-682. Dol: http://dx.doi.org/10.1353/csd.0.0098

* Rojas, M. (2009) El abandono de los estudios. Deserción y decepción de la juventud. Hologramática, vi(10), v4, 75-94. http://cienciared.com.ar/ra/usr/3/467/ hologramatica_n10_vol4pp75_94.pdf

* Rovella, A., y Sans, M. (2005). Predictores de éxito académico en estudiantes universitarios. Revista Electrónica de Psicología Política, 2(6), 1-5.

- Saavedra Guajardo, E., y Villalta Paucar, M. (2008). Medición de las características resilientes, un estudio comparativo en personas entre 15 y 65 años. LIBERABIT, 14, 31-40. http://www.scielo.org.pe/pdf/liber/v14n14/a05v14n14

- Salanova Soria, M., Martínez Martínez, I., Bresó Esteve, E., Llorens Gumbau, S., y Grau Gumbau, R. (2005). Bienestar psicológico en estudiantes universitarios. Facilitadores y obstaculizadores del desempeño académico. Anales de Psicología, 21(1), 170-180. https://www.redalyc.org/articulo.oa?id=167/16721116

* Seminara, M. P., y Aparicio, M. (2018). Deserción universitaria. ¿Un concepto equivoco? Revisión de estudios latinoamericanos sobre conceptos alternativos. Revista de Orientación Educacional, 32(61), 44-72. https://dialnet.unirioja.es/servlet/ articulo?codigo $=6739300$

* Stelnicki, A., Nordstokke, D., y Saklofske, D. (2015) Who is the successful university student? An analysis of personal resources. Canadian Journal of Higher Education, 45(2), 214-228. http://journals.sfu.ca/cjhe/index.php/cjhe/article/view/184491

* Valle, G., Raiano, J., García, S., Suligoy, M., y Gómez, G. (2014). Éxito y fracaso académico desde la perspectiva de los estudiantes universitarios. Dos Puntas, vi(9), 161-186. https://dialnet.unirioja.es/descarga/articulo/5067154.pdf

\section{Cómo CITAR ESTE ARTículo}

* Seminara, María Paula (2020, septiembre-octubre). La deserción universitaria: resiliencia como posibilidad de logro. Revista Digital Universitaria (RDU), 21(5). DOI: http://doi.org/10.22201/cuaieed.16076079e.2020.21.5.11 